\title{
Performance Comparison of Various Hierarchical WSN Routing Protocols
}

\author{
Hamdy H. El-Sayed1 \\ 1 faculty of computers \& Information ,Sohag University, Egypt. \\ Hamdy2006x@gmail.com \\ Hassan Shaban Hassan ${ }^{2}$ \\ 2 faculty of computers \& Information , Minia University, Egypt. \\ hassan_sh_ha@yahoo.com
}

ABSTRACT

Wireless Sensor Networks (WSN) is composed of small sensor nodes which may be hundreds or multi hundreds or thousands in number. Sensor nodes, also known as mote, are small, lightweight and portable devices equipped with a transducer, microcomputer, transceiver, and power source. Based on the network topology, routing protocols in sensor networks can be classified as flat-based routing, hierarchical-based routing and location-based routing. This paper studied some hierarchical-based routing protocols and evaluated these protocols with different cluster head probability in medium network with 200 nodes number. Protocols like Low Energy Adaptive Clustering Hierarchy (LEACH), Distributed Energy-Efficient Clustering (DEEC), Threshold sensitive Energy Efficient sensor Network protocol (TEEN) and Stable Election Protocol (SEP) are used for our comparisons. We evaluate the performance of these protocols for a cluster head probability sensing application. Cluster head Probability effects on throughput, overhead, packet delivery ratio, alive nodes and dead nodes, as parameters used to measure the performance of these protocols. We observed new results and different comparisons for hierarchical protocols in WSN.

Keywords - alive nodes, dead nodes, packet delivery ratio, throughput.

\section{INTRODUCTION}

$\mathrm{W}$ which have capabilities of communication. Wireless sensor nodes have little period amount of battery power and this is the major issue in wireless sensor networks. WSN component of hundreds or even thousands of sensors nodes which are randomly deployed in the area of research [1]. Sensor nodes sensed physical data and send it to base station. These nodes are capable of communicate with each other for sending and receiving information either directly or through other intermediate nodes, and for that each node in the sensor network work as a router inside the network. Sensor nodes can be found in long distance from base station, so it is superfluous to send this data to base station due to limited energy in the nodes. Clustering can be used to reduce the energy consumption. The basic idea of hierarchical routing protocols is to cluster sensor nodes and communication within a cluster using cluster head nodes. Cluster head nodes can gather and integrate data to reduce the transmission amount, and finally the cluster head node can send integrated data to the sink node. This mode can meet the scalability of the sensor network and maintain the energy consumption of the sensor node to prolong the life cycle of the network [2].

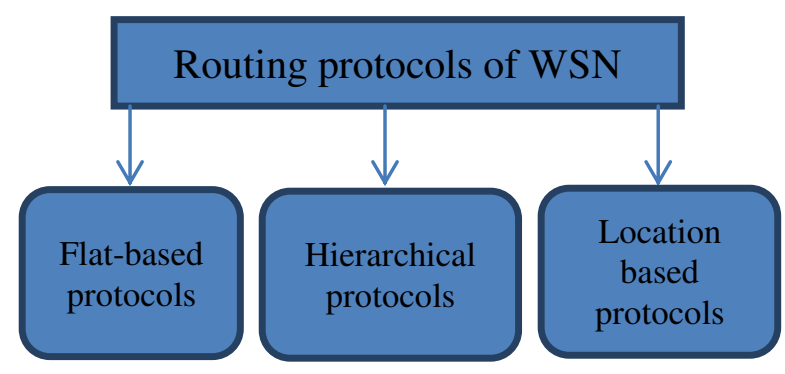

Fig. 1. Routing protocols classification of WSN In any network especially wireless sensor networks, for efficient performance; its protocols must be very effective. Different protocols are developed that address power problem in sensor networks. Most important routing algorithms can be categorized into three types like direct transmission algorithms, hop to hop transmission algorithms and cluster based algorithms. Another problem that persists is to handle bulk of information sensed and passed over by every node of a network [3]. In an efficient wireless sensor network, we need efficient routing protocol that has low routing overhead and well organized data aggregation mechanisms to increase efficiency of network and to save the limited power of sensor node. We discuss about the work done on hierarchical routing protocols of wireless sensor networks along with some changes of cluster head probability. Some different parameters have been used like overhead, packet delivery ratio, throughput, dead nodes number and alive nodes. Finally, experiments along with comparisons are made on some hierarchical routing protocols in WSN with the 
parameters that have been used and obtained different new result that discussed briefly. This paper has been organized as follows in section 1: an introduction on the article. Section 2: relation work. Section 3: discusses the simulation and results. Section 4: discusses the conclusion. Lastly the references presented in section 5 .

\section{2- WNS ROUTING PROTOCOLS}

\section{A. DEEC}

In DEEC, the cluster-heads are elected by a probability due to the ratio between residual energy of each node and the average energy of the network. The epochs of being cluster-heads for nodes are different according to their initial and residual energy. The nodes with high initial and residual energy have chances to be the cluster-heads more than the nodes with low energy [4]. DEEC [5] protocol is developed for heterogeneous network which have three types of different initial energy of nodes with weighted different probability for electing CHs.

\section{B. $\mathrm{LEACH}$}

Low-energy adaptive clustering hierarchy (LEACH) [6] protocol is a classical protocol. Clustering is an efficient ethod to handle scalability problem and energy consumption challenge is promising in improving the scalability and extending the lifetime of WSNs. For this reason, it is widely exploited in WSN applications [7].

\section{SEP}

Stable Election Protocol (SEP) [8] is a heterogeneousaware protocol to prolong the stability period, which is crucial for many applications where the feedback from the sensor network must be reliable. SEP is based on weighted election probabilities of each node to become cluster head according to the remaining energy in each node. By studying the sensitivity of SEP protocol to heterogeneity parameters capturing energy imbalance in the network. SEP yields longer stability region for higher values of extra energy brought by more powerful nodes.

\section{TEEN}

Threshold Sensitive Energy Efficient Sensor Network Protocol (TEEN) [9]. The sensor network architecture in depends on various hierarchical clustering. TEEN is information driven, reactive, event driven convention which is most appropriate for time basic application. It transmits information in hard threshold and soft threshold values. If the thresholds that are not achieved, then nodes will never communicate

\section{3- RELATED WORK}

To pursue the effective routing protocols for WSNs, many researchers have done lots of studies recently and got the result that a scheme with hierarchy and clustering. [10] Showed that as the cluster head percentage increases the number of live nodes decreases but increment at $8 \%$ and after that decreases again. Also as the cluster head number increases, the energy consumption increases except for $8 \%$ it decreases, but on overall the energy consumption increased. Paper [11] showed that the total energy consumption of the network is decreased by prioritizing which maximize the network lifetime. Paper [12] analyzed the energy consumption, delay and traffic bandwidth for some cluster based routing protocols such as $\mathrm{LEACH}$, CBHRP, MH-LEACH and LEATCH. The CBHRP protocol has the best results.

\section{4- SIMULATION AND RESULTS}

Some homogenous protocols like LEACH and TEEN and heterogeneous protocols like SEP and DEEC of WSN have been simulated using MATLAB 2016a and the parameters are listed in the table 1. Also, we are used medium network with 200 number of network nodes to test the performance of these protocols. Also, probability of cluster head changes with medium network has been used for testing. The parameters that have been used to test the comparison are the number of dead and alive nodes, throughput, overhead and packet delivery ratio in WSN network.

TABLE I

LIST OF SIMULATION PARAMETERS

\begin{tabular}{lcc}
\hline \hline No. & Parameters & Values \\
\hline 1 & Network Area & $300 * 300$ \\
2 & Number of nodes, & 200 \\
3 & Cluster head & $0.3,0.03$ \\
& probability p & $50 * 0.000000001$ \\
4 & transmitter energy & $50^{*} 0.000000001$ \\
5 & receiver energy & $5^{*} 0.000000001$ \\
6 & Aggregation Energy & $0.0013 * 0.000000000001$ \\
7 & amplification & 5000 \\
8 & energy & 100 \\
9 & Number of Rounds & 2 \\
10 & Hard Threshold & \\
& Soft Threshold & \\
\hline \hline
\end{tabular}

\section{E. Performance analysis with cluster head probability 0.3}

First simulation purpose, 200 nodes and 5000 rounds with probability cluster head 0.3

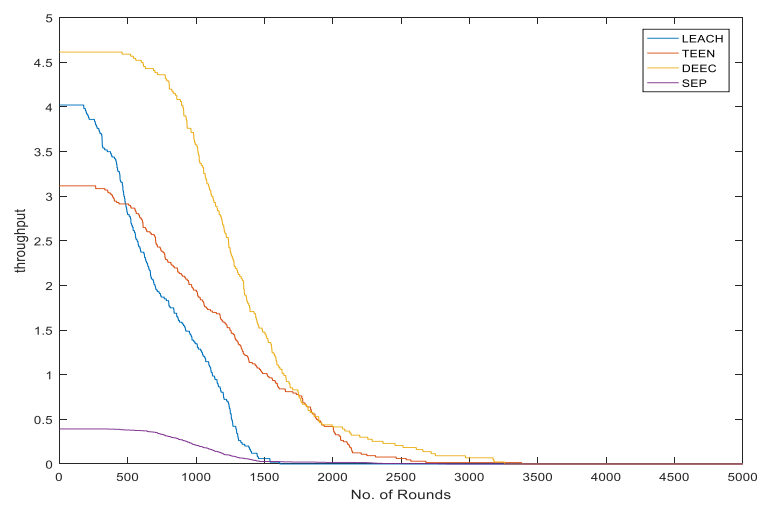

Fig. 2. Shows throughput with 0.3 probability 
As shown in figure 2 all protocols throughput decreased with increased number of rounds. But Throughput of DEEC is the maximum other than protocols and LEACH is the second maximum put when reached 500 rounds we found that TEEN exceeds than LEACH. SEP protocol throughput is less than other protocols.

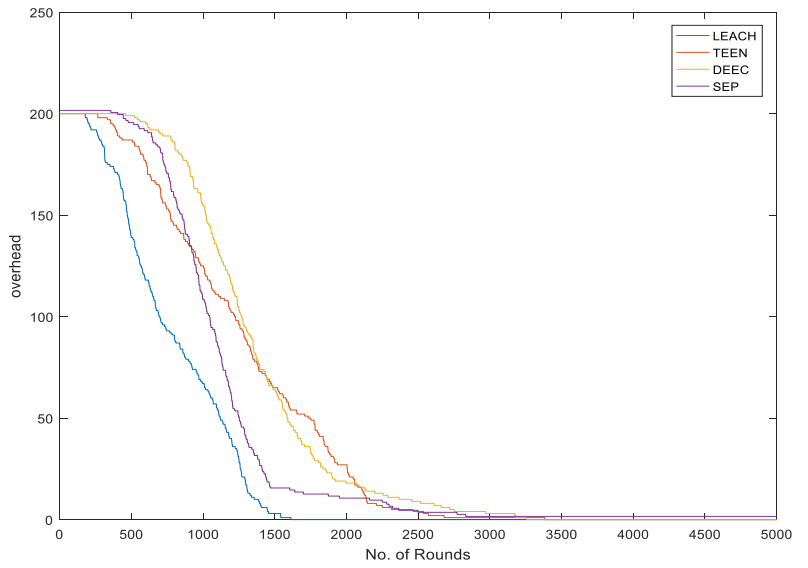

Fig. 3. Shows overhead with 0.3 probability.

Also in figure 3 overhead which mean "routing and data packets have to share the same network bandwidth most of times and hence routing packets are considered to be overhead in the network" [ 13] of all protocols decreased with increasing number of rounds but Overhead of LEACH protocol is less other than protocols and DEEC have maximum overhead. TEEN protocol overhead exceeds than SEP in 1100 rounds and they reached the same over head when reached 2500 rounds.

In Figure 4 the packet delivery ratio of all protocols are increased with increasing the number of rounds. Packet delivery ratio of LEACH exceeds than others and reaches the maximum in 1500 rounds after that are stable. TEEN and DEEC exceeds in the same manner and reaches the maximum in 2000 rounds. The packet delivery ratio for SEP protocol is less than other protocols and also reaches the maximum in 1500 rounds after that stable after that.

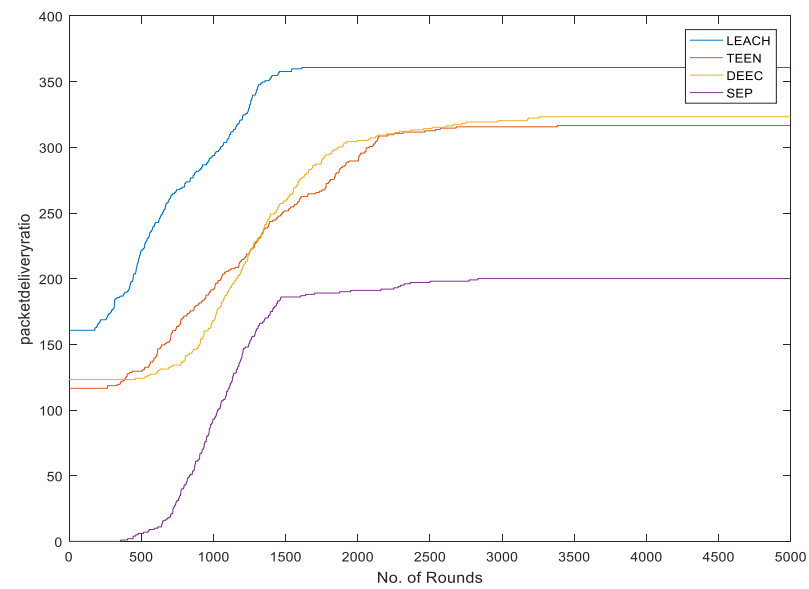

Fig. 4. Depicts packet delivery ratio for protocols with 0.3 probability.
Figure 5 shows that the network lifetime decreased with increasing number of rounds LEACH protocol life time less than other protocols until 1500 rounds. SEP protocol exceeds than TEEN and by increasing number of rounds TEEN exceeds than SEP in 1000 rounds and exceeds than DEEC when reaches 1500 and less from it when reaches 2100 rounds. Network life time still 3250 rounds of network.

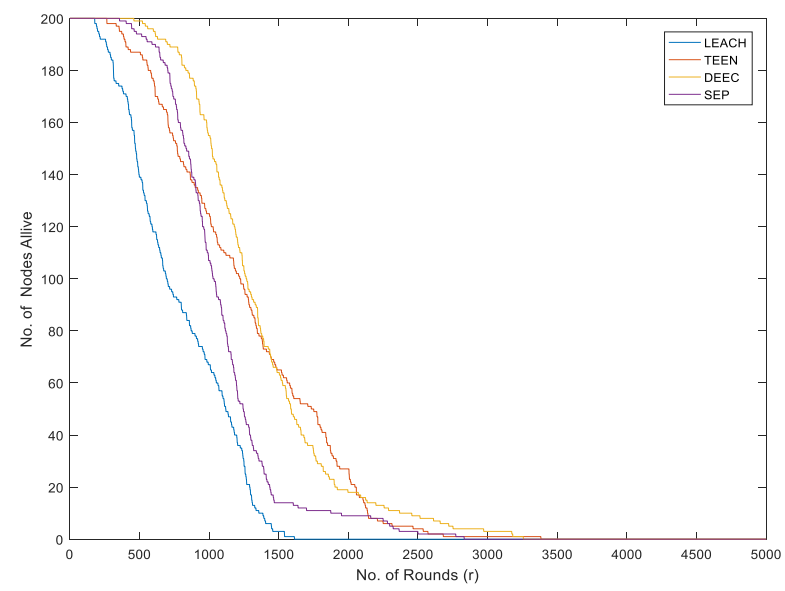

Fig. 5. Depicts alive nodes for protocols with 0.3 probabilities.

Figure 6 shows that all dead nodes exceeds with increasing number of round. Dead nodes number of LEACH less than other protocols. LEACH protocol dead nodes number reaches maximum in 1500 rounds and the other protocols dead nodes number reach maximum in 3000 rounds.

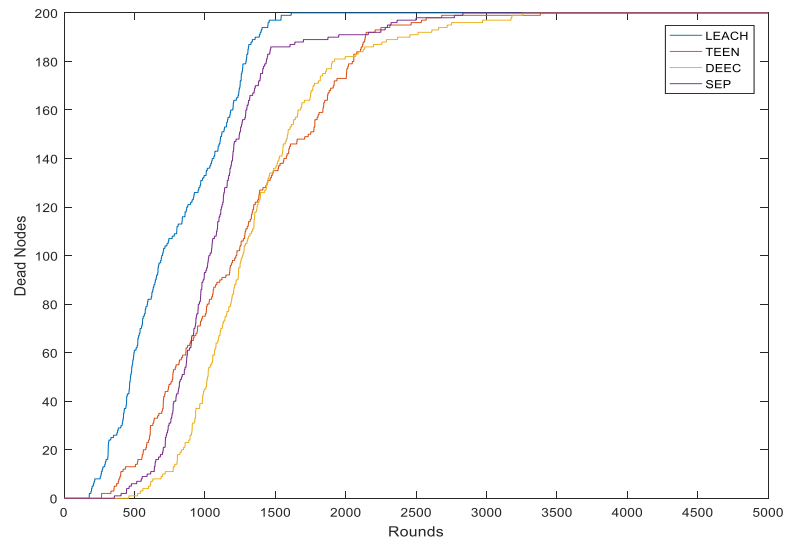

Fig. 6. Dead nodes with 0.3 probability.

\section{F. Performance Analysis cluster head with probability 0.03}

Second simulation with 200 nodes and the number of rounds 5000 with probability cluster head 0.03 .

As shown in figure 7 all protocols throughput decreased with increased number of rounds. But Throughput of LEACH is the maximum other than protocols and TEEN is the second maximum put when reached 500 rounds we 
found that DEEC less than previous protocols. The throughput of SEP protocol is less than other protocol. The Throughput of protocols increased with decreased cluster head probability.

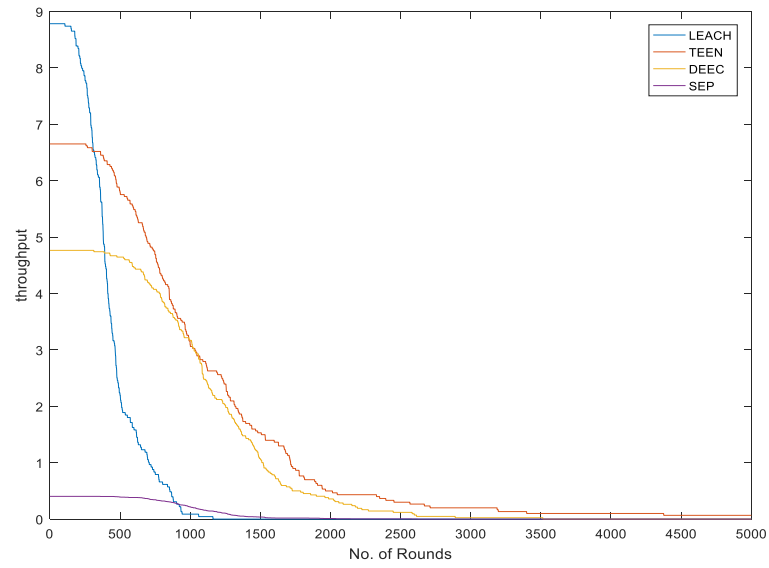

Fig. 7. Shows throughput with 0.03 probability

Figure 8 shows that the overhead of all protocols decreased with increasing number of rounds but Overhead of LEACH protocol is less other than protocols and DEEC have maximum overhead. SEP protocol overhead exceeds than TEEN until reached 1100 rounds and DEEC and SEP reached the same over head when reached 2500 rounds. But overhead of TEEN reaches at 3500 rounds.

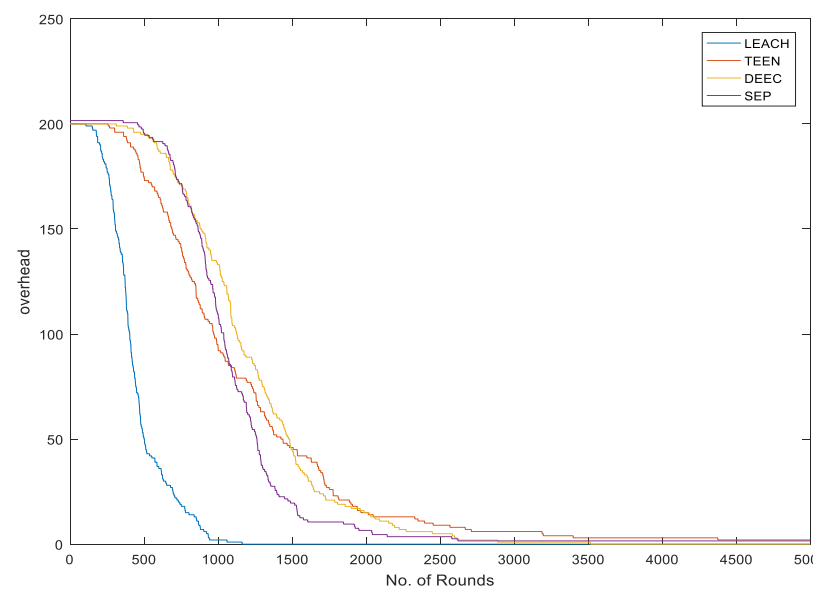

Fig. 8. Depicts overhead with 0.03 probability.

As shown in figure 9 all packet delivery ratio values increased with increasing number of rounds. Packet delivery ratio of DEEC protocol exceeds the others and reaches the maximum in 400 rounds. LEACH exceeds than DEEC until 1500 rounds then DEEC exceeds than other protocols. TEEN and SEP protocols exceeds in the same manner and reaches the maximum in 1500 rounds. SEP protocol packet delivery ratio values less than others and also reaches the maximum in 1500 rounds than TEEN and be stable after that. Also the manner of protocols that has been observed is different with changing decreasing cluster head probability.

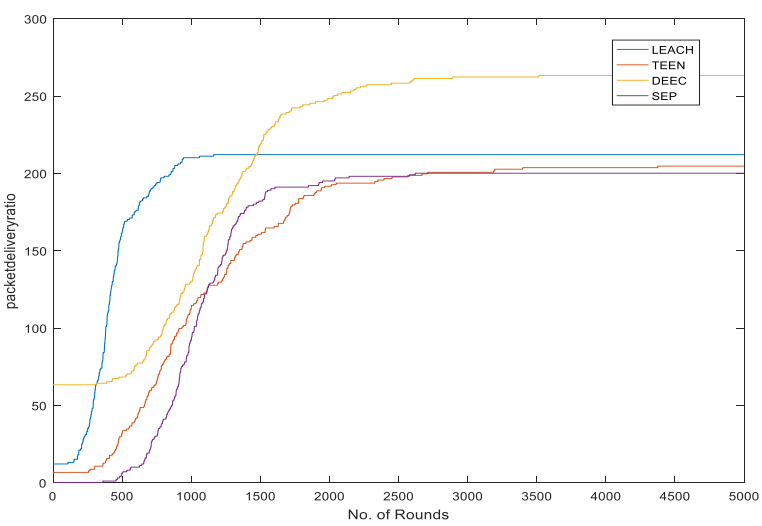

Fig. 9. Packet delivery ratio for protocols with 0.03 probability.

Network lifetime decreased with increasing number of rounds as in figure 10. Also LEACH protocol life time less than other protocols. SEP and DEEC protocols life time exceeds than TEEN and by increasing number of rounds TEEN and DEEC life time exceeds than SEP in 1200 rounds. Network life time of LEACH decreased with decreased cluster head probability but other protocols lifetime TEEN, DEEC and SEP increased with decreased cluster head probability.

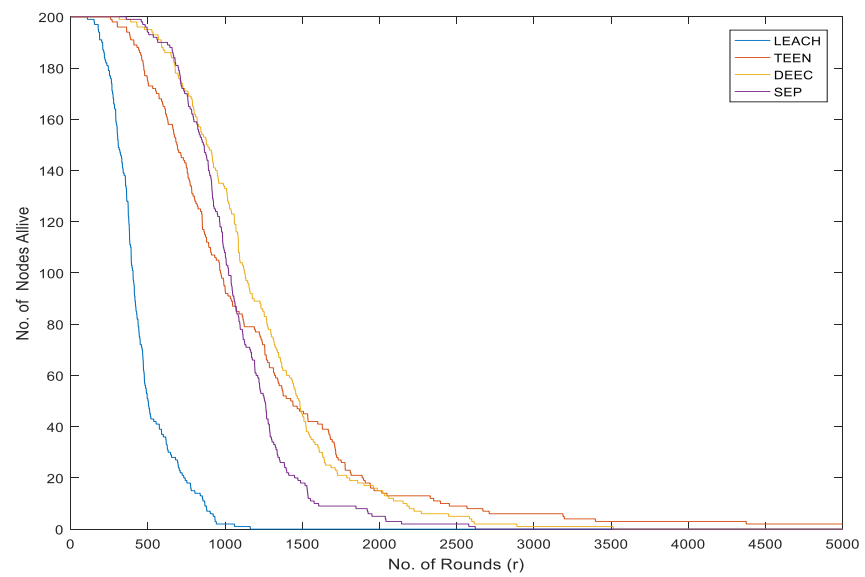

Fig. 10: Depicts alive nodes for protocols with 0.03 probability.

Figure 11 shows that dead nodes exceed with increasing number of round. Dead nodes number of LEACH less than of other protocols. Dead nodes number reaches maximum in 1000 rounds for LEACH protocol. The other two protocols DEEC and SEP the dead nodes number reach maximum in 2500 rounds. But in TEEN protocol dead nodes number reaches the maximum in 3500 rounds. We observed that the dead nodes numbers are different with changing cluster head probability 


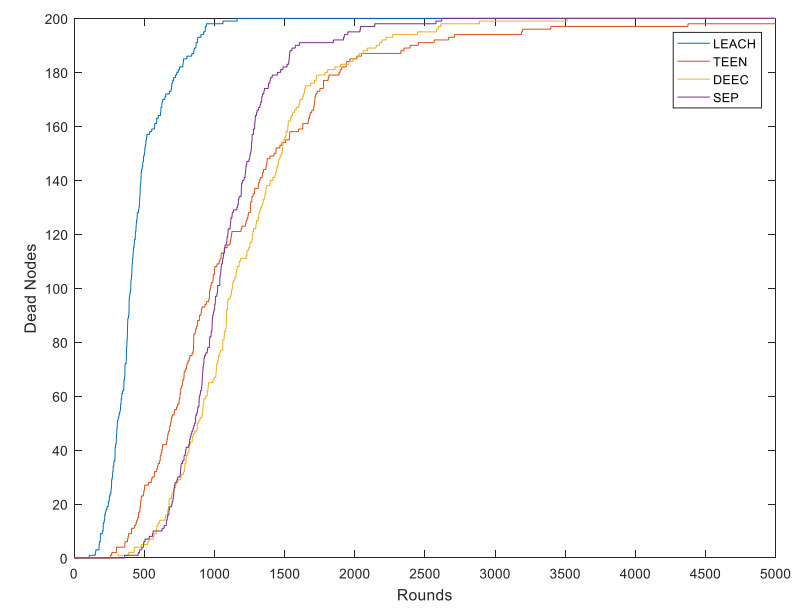

Fig. 11. Dead nodes number for protocols with 0.03 probability.

TABLE 2.

COMPARISON OF SOME DIFFERENT HIERARCHICAL BASED ROUTING PROTOCOLS

\begin{tabular}{ccccc}
\hline Parameters & LEACH & TEEN & DEEC & SEP \\
\hline $\begin{array}{c}\text { Energy } \\
\text { efficiency } \\
\text { Data } \\
\text { aggregatio }\end{array}$ & Very high & Good & High & $\begin{array}{c}\text { Moderat } \\
\text { e }\end{array}$ \\
n & Yes & Yes & Yes \\
Network & $\begin{array}{c}\text { Decrease } \\
\text { d with }\end{array}$ & $\begin{array}{c}\text { increased } \\
\text { with } \\
\text { Lifetime } \\
\text { decreased }\end{array}$ & $\begin{array}{c}\text { increased } \\
\text { with } \\
\text { decreased }\end{array}$ & $\begin{array}{c}\text { increase } \\
\text { d with } \\
\text { decrease }\end{array}$ \\
Scalability & No & No & p & d p \\
Type of & Fixed & Fixed & Fixed & No \\
network & Finged \\
Hope & Single & Single & Single & Single \\
count & hope & hope & hope & hope \\
Mobility & Fixed BS & Fixed BS & Fixed BS & Fixed \\
Over head & High & High & Very high & High \\
Power & High & High & High & High \\
Usages & No & No & No & No \\
QoS & No & & &
\end{tabular}

\section{5- CONCLUSION}

From the above results that has been obtained by varying the cluster head probability while transmitting the data over the network and analyzing its various performance metrics with different parameters of routing protocols the results showed that the throughput, overhead, packet delivery ratio, alive nodes and dead nodes number are effected and differentiate with changing the cluster head probability. As regards to the cluster head probability for hierarchical protocols we found different results of using deferent parameters to test the performance of these protocols. Therefore the network lifetime different with different cluster head probability as compared to these protocols.

\section{Future Contribution}

In future many other techniques should be developed which should focus on improving the network performance so that the lifetime as well as the performance of the wireless senor networks can be improved. The hierarchical routing protocols have been affected in its performance as well as cluster head probability changes and the different parameters that have been used proved that. The adaptability of the protocols can be checked out and they even can be made more flexible to all kinds of applications as well as environments from time to time.

\section{6- REFERENCES}

[1] I.F. Akyildiz, W. Su, Y. Sankara subramaniam and E. Cayirci, "Wireless sensor networks: a survey, Computer Networks", 38 (4) (2002) 393-422.

[2] Lei An 1, Aihua Li 1, Ling Pang2 and Guangdong He1 : "Analysis and Comparison of Routing Protocol of Wireless Sensor Network", iJOE - Volume 12, Issue 10, 2016.

[3] D. Mahmood, N. Javaid, S. Mahmood, S. Qureshi, A. M. Memon, and T. Zaman, " MODLEACH: A variant of LEACH for WSNs", in Proc. 8th Int. Conf. Broadband Wireless Comput., Commun. App.(BWCCA), pp. 158-163, Oct.2013.

[4] L. Qing, Q. Zhu, M. Wang, "Design of a distributed energy efficient clustering algorithm for heterogeneous wireless sensor network", ELSEVIER, Computer Communications 29, pp 2230- 2237, 2006.

[5] Li Qing, Qingxin Zhu, Mingwen Wang, Design of a distributed energy-efficient clustering algorithm for heterogeneous wireless sensor networks, Computer Communications, Volume 29, Issue 12, 4 August 2006, Pages 2230-2237, ISSN 0140-3664.

[6] W. B. Heinzelman, A. P. Chandrakasan, and H. Balakrish-nan, "An application-speciic protocol architecture for wireless microsensor networks," IEEE Transactions on Wireless Communications,vol.1,no.4,pp.660-670,2002.

[7] C. Sevgi and A. Kocyigit, "On determining cluster size of ran-domly deployed heterogeneous WSNs," IEEE Communications Letters, vol.12, no.4,pp.232234,2008

[8] G. Smaragdakis, I. Matta, A. Bestavros,"SEP: A Stable Election Protocol for clustered heterogeneous wireless sensor networks." in: Second International Workshop on Sensor and Actor Network Protocols and Applications (SANPA 2004), 2004. 
[9] Tamanna and Anshu Sharma: " Analyze and implementation of TEEN Protocol in Wireless Sensor Network", International Journal of Innovative Research in Science, Engineering and Technology, Vol. 5, Issue 3, March 2016.

[10] Amita Yadav and Suresh Kumar "Analysis and Simulation of Low-Energy Adaptive Clustering Hierarchy Protocol" Advances in Intelligent Systems and Computing, Volume 638, Proceedings of CSI2015.

[11]Ozair Ahmad 2015, 'Design and Evaluation of Clustering Protocol to Enhance the Lifetime of WSN', International Journal of Advanced Technology in Engineering, and Science, vol. 3, no. 4, pp. 23482755.

[12] Mariam Benmoussa, Mariyam Ouaissa et.al., ,'QoS Analysis of Hierarchical Routing Protocols for Wireless Sensor Networks', In the Proceedings of the Second International Conference on Internet of Things, Data and Cloud Computing, ACM Digital Library, A.No.89, 2017.

[13] Hamdy H. El-Sayed: "Performance comparison of LEACH, SEP and Z-SEP Protocols in WSN", International Journal of Computer Applications (0975 - 8887) Volume 180 - No.30, April 2018.

\section{Biographies and Photographs}

\section{First author}

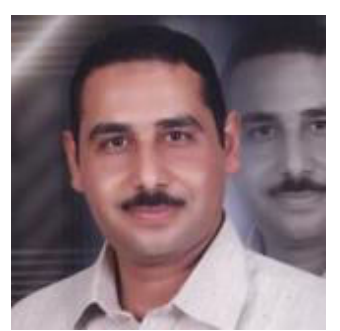

Hamdy H. El-Sayed received the $\mathrm{PhD}$ degree in wireless ad hoc network routing protocols from computer science department sohag university egypt march ,2015 . His research interests are in the areas of ad hoc routing protocols and sensor networks, IoT,cloud computing and security.

\section{Second author}

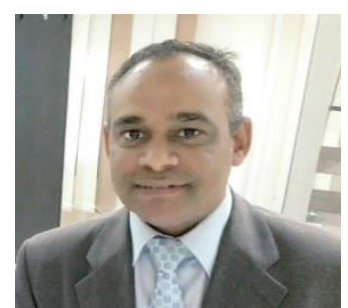

Hasssn Shaban received his Ph.D. degree in Computer Science. Currently work as lecturer at Computer Science Dept., Faculty of Computers and Information, Minia University, Egypt. His research interests include wireless sensor networks, security, IOT, Machine learning, optimization, metaheuristics. 